\title{
FREQUENCY SPECIFIC AND NONSPECIFIC EFFEGTS OF FLICKERING LIGHTS UPON ELECTRICAL ACTIVITY OF THE HUMAN OCCIPUT ${ }^{1}$
}

\author{
SHINKURO IWAHARA, SETSUKO NOGUCHI, KUO-MAN YANG \\ AND HIROSHI OISHI
}

Department of Psychology, Tokyo University of Education

\begin{abstract}
The occipital EEG was observed under the rhythmic photic stimulation (F) at $7.5,9.2,11.4,15.0$ and $22.5 \mathrm{~Hz}$ as well as under the nonflicker (NF) stimulation for 11 human adults. The data were frequency-analyzed with a Waltertype apparatus and the obtained integrated values in 10-sec units for 7 frequency bands (theta 1 and 2, alpha 1,2 and 3 , beta 1 and 2) were used as cortical responses. The spontaneous or NF activity was blocked by eye opening (EO) for all bands, particularly for the alpha band in which the $S$ 's spontaneous alpha activity was included. The same blocking effect (I) was observed by flicker both with eyes closed and opened, and this effect was relatively independent of flicker frequency. Thus, the nonspecific $I$ effect was estimated per band, and the frequency-specific effect (FE) was computed by additing the absolute value of $I$ to the difference in the cortical response between $F$ and $N F$; that is, $F E=(F-N F)$ + I. The obtained FE spectrum in relation to the NF spectrum was little affected by EO, was only a little different, if any, between the dominant alpha and the poor alpha subjects, and had a wider distribution with a higher mode (alpha 3 rather than alpha 2 ).
\end{abstract}

As is well known, light stimulation can block the alpha wave activity, recorded predominantly from the human occiput, but if it is given intermittently, it can also drive brain rhythms not very different in frequency from the spontaneous or basic alpha activity. The former is called "alpha blocking", which is nonspecific in the sense that the effect is produced by various stimuli even in different modalities while the latter is called " photic driving", which is specific in the sense that the driven rhythm follows or tunes the stimulus frequency and often its harmonics as well. These two phenomena have been usually treated independently (e.g., Morgan, 1965).

Our recent study (Noguchi \& Iwahara,

1 Requests for reprints should be sent to Shinkuro Iwahara, Department of Psychology, Tokyo University of Education. 3-29-1 Otsuka, Bunkyoku, Tokyo 112, Japan.
1971) suggested that this traditional view must be somewhat modified. First, the blocking effect due to eye opening (EO) is not restricted to the alpha bands (8-9, 9-11 and $11-13 \mathrm{~Hz}$ ) but it is also observed in the beta (13-20 and $20-30 \mathrm{~Hz}$ ) and theta (4-6 and 6-8 Hz) band, although the effect is strongest in the alpha bands, especially in the donimant alpha frequency. Second, flicker stimulation also produces a marked reduction in the basic alpha activity. Based on these findings, it was assumed that flicker stimulation has both the frequency-specific facilitative effect (FE) and the frequency-nonspecific inhibitory effect (I) upon the occipital response and as a first approximation the observed cortical response is a linear function of these antagonistic effects. Here, two points must be understood. First, the cortical response was measured in terms of integrated values per $10 \mathrm{sec}$ with bandpass filters, and similar results 
were obtained if the integrated values were squared. Second, the terms, "facilitation" and "inhibition" were used simply to indicate "increases" and "decreases", respectively in the gross cortical response.

This study aimed to evaluate quantitatively the frequency-specific effect of each of the 5 flickering lights of different frequencies either with eyes-closed (EC) or eyes-opened (EO) and to investigate the relationships between the frequencyspecific effect and the basic EEG pattern.

\section{METHOD}

Subjects. Subjects were 11 healthy adults of both sexes whose ages ranged from 20 to 47 years; they all possessed the normal EEG patterns.

Apparatus. The EEG was recorded by a 13-channel ink-writing electroencephalograph (San-ei model EG-130) from the frontal, vertex and occipital needle electrodes with a reference electrode attached to the right earlobe. However, only the occipital EEG was processed with a Walter-type frequency analyzer (San-ei model EA-201), which produced an integrated value per $10 \mathrm{sec}$ for each of the following frequency bands: delta $1(1-2 \mathrm{~Hz})$, delta 2 (2$3 \mathrm{~Hz}$ ), delta $3(3-4 \mathrm{~Hz})$, theta $1(4-6 \mathrm{~Hz})$, theta $2(6-8 \mathrm{~Hz})$, alpha $1(8-9 \mathrm{~Hz})$, alpha $2(9-11$ $\mathrm{Hz}$ ), alpha $3(11-13 \mathrm{~Hz})$, beta $1(13-20 \mathrm{~Hz})$ and beta $2(20-30 \mathrm{~Hz})$. Because of possible artificial effects on the slower activities, the integrated values for the 3 delta bands were not analyzed in this study.

Flicker stimulation was given at 7.5, 9.2, $11.4,15.0$ and $22.5 \mathrm{~Hz}$ of the same intensity, being generated by a photic stimulater (Nihonkoden model MS-1PS).

Procedure. The subject lay abed in a dark, sound-proofed, electrically-shielded room and the EEG was taken monopolarly as described above together with EKG and GSR. Following the recording of the basic, that is, nonflicker (NF) EEG both with EC and EO, the subject was exposed to each of the 5 flickering lights for a total of at least three 10 -sec units with both EC and EO. Order of flicker presentation per unit, was randomized as much as possible with a few NF units in between. After all combinations of flicker combinations were completed, the basic or NF EEG was again taken for about 5 units. Results indicated no obvious differences in the basic EEG patterns between the first and the last 5 units either by inspection or by integrated values and thus the background cortical activity may be assumed to be constant throughout the experiment during which the subject was required to be alert. The experiment was concluded in about $1 \mathrm{hr}$.

\section{Results}

Basic EEG patterns. As in our previous studies, the cortical activity was measured in terms of integrated values with bandpass filters. Fig. 1 indicates the mean integrated values of the 5 different frequency bands with EC and EO across 11 subjects, where no flicker (NF) was presented. For brevity, this figure is called the NF spectrum or the basic spectrum.

Analysis of variance of the data yielded a significant $F$ with regard to the overall EC-EO difference $(F=28.61, \quad d f=1 / 10$, $p<0.001)$, as well as to the overall band difference $(F=7.01, d f=6 / 10, p<0.001)$ and to the interaction between the two effects $(F=9.29, d f=6 / 60, p<0.001)$. By the use of individual $t$ tests, the EC-EO difference or the nonspecific inhibitory effect was significant for all bands except for theta 1 .

When the total 11 subjects were divided into 6 slow alpha subjects whose basic alpha belonged to alpha 2 and into 5 fast alpha subjects whose basic alpha belonged to alpha 3 , the overall EC-EO difference was small and was short of statistical significance $(F>1, d f=1 / 9)$, as shown in Table 1. However, there was a significant interaction between the group and band variables $(F=7.09, d f=$ $1 / 9, p<0.05)$ in which the alpha 3 blocking was largest for the fast alpha group, while 
TABLE 1

Decreases in occipital integrated values by eye-opening (EO) for the fast and slow alpha subjects

\begin{tabular}{|c|c|c|c|c|c|c|c|c|c|}
\hline \multirow{3}{*}{ Group } & \multirow{3}{*}{$\mathcal{N}$} & \multicolumn{7}{|c|}{ Frequency bands } & \multirow{3}{*}{ Total } \\
\hline & & \multicolumn{2}{|c|}{ theta } & \multicolumn{3}{|c|}{ alpha } & \multicolumn{2}{|c|}{ beta } & \\
\hline & & 1 & 2 & 1 & 2 & 3 & 1 & 2 & \\
\hline Fast alpha & 5 & -0.4 & 1.4 & 1.6 & 9.0 & 13.2 & 5.4 & 3.4 & 33.6 \\
\hline Slow alpha & 6 & 1.9 & 3.7 & 6.2 & 19.8 & 5.5 & 3.8 & 1.8 & 42.7 \\
\hline Difference & & -2.3 & -2.3 & -4.6 & -10.8 & 7.7 & 1.6 & 1.6 & -9.1 \\
\hline
\end{tabular}

Note: The total 11 subjects were divided into 5 fast alpha subjects whose basic alpha response belonged to the alpha 3 band $(11-13 \mathrm{~Hz}$ ) and 6 slow alpha subjects whose basic alpha belonged to the alpha 2 band $(9-11 \mathrm{~Hz}$ ). As shown in the table, blocking by eye opening (EO) was largest in the frequency band in which the subject's own basic alpha frequency was included. Similar results were obtained by flicker stimulation as shown in Text.

the alpha 2 blocking was largest for the slow alpha group.

Differences between ficker and nonficker responses $(F-\mathcal{N} F)$. Fig. 2 shows sample recordings from the parietal and occipital cortices with EC and EO without flicker (NF) and with flickers (F) at 9.2 and $11.4 \mathrm{~Hz}$ for Subject IW. On the left side of the figure are plotted the occipital integrated values for 7 frequency bands and the differences between $\mathrm{F}$ and $\mathrm{NF}$ or (F-NF). The band in which the flicker frequency was included is indicated by an arrow. The means of such F-NF scores across 11 subjects are depicted in Fig. 3. This F-NF score is assumed in this study

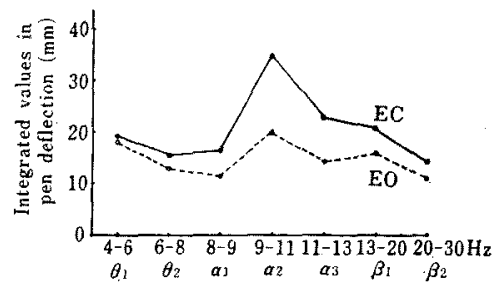

Fig. 1. Spontaneous cortical responses in integrated values per 10 -sec epoch from the monopolar occipital electrode for 7 frequency bands based on 11 healthy human adults. EC=eyes closed, EO=eyes opened. as a linear function of the frequency specific effect plus the frequency nonspecific effect. This is merely a first approximation and may be modified in a future study. As shown in Fig. 3, the difference scores (F-NF) were very similar between $\mathrm{EC}$ and EO. As a matter of fact, the overall EC-EO difference was not significant for any of the 5 flickers and the interaction between the band and EC-EO effects was significant only for flicker at $9.2 \mathrm{~Hz} \quad(F=2.80, d f=6 / 60, p<0.05)$, in which the F-NF score was clearly less with $\mathrm{EC}$ than EO.

The F-NF score was always negative for the alpha 2 band, except the case with flicker at $9.2 \mathrm{~Hz}$ which is included in that band, although the score was small even in this case. In addition, this F-NF score was relatively independent of flicker frequency outside alpha 2 and 3 (i.e., excepting flicker at 9.2 or $11.4 \mathrm{~Hz}$ ). That is, flicker stimulation had the frequency nonspecific blocking effect. Although the effect appears less marked for alpha 3 band than for alpha 2 band, the alpha 3 blocking was evident when the slow alpha subjects were eliminated.

Fig. 3 also suggests that the F-NF score was positive not only at flicker frequency 
but also at its harmonics excluding the subharmonics. For example, flicker at $11.4 \mathrm{~Hz}$ produced a positive $\mathrm{F}-\mathrm{NF}$ score for the tuned (alpha 3) and the harmonic (beta 2) band but not for the subharmonic (theta 1) band.

Frequency-specific effect. Fig. 4 indicates the F-NF scores with arrows in Fig. 2 or the increased integrated values of the bands specific to flicker stimulation.
Analysis of variance of the data produced a significant $F$ only for the band (flickerfrequency) effect $(F=4.04, d f=4 / 40, p<$ $0.01)$. Even when the total subjects were divided into the dominant and the poor alpha subjects, the difference between the 2 groups was small and nonsignificant (Fig. 4).

As was assumed above, flicker stimulation produces not only the frequency specif-

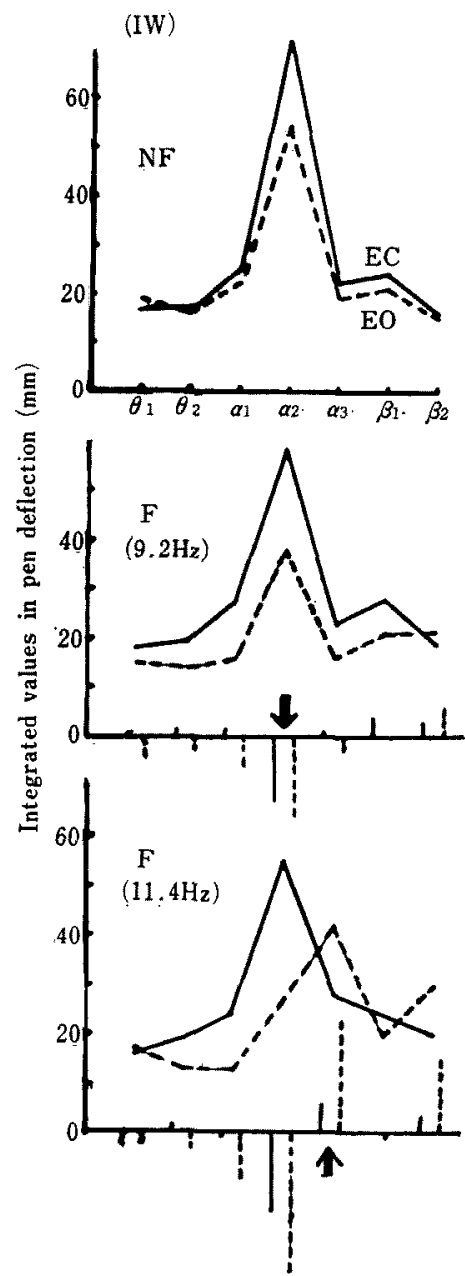

EC (Eyes Closed)

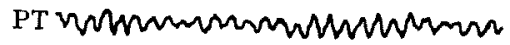

occurnmanumburmons

EO (Eyes Opened)

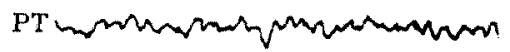

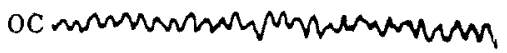

EC.

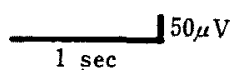

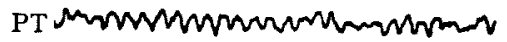

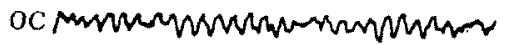

EO

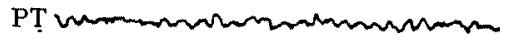

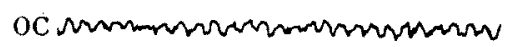

EC

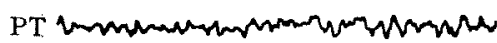

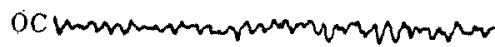

EO

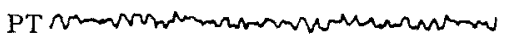

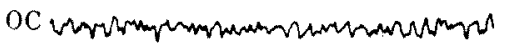

FIG. 2. Sample recordings from the parietal (PT) and the occipital (OC) cortices with and without flicker. On the left side are plotted the integrated values from the occipital leads for 7 frequency bands and the differences in occipital integrated values between fiicker (F) and nonflicker (NF) conditions. Arrows indicate the band in which the flicker frequency was included. 
ic driving effect (FE) but also the frequency nonspecific blocking effect (I) and the observed F-NF score is a sum of the two effects or $(\mathrm{F}-\mathrm{NF})=\mathrm{FE}-\mathrm{I}$. In this study, the I effect was tentatively estimated in terms of the largest NF minus F score. For example, in case of Subject IW with EC, the F-NF was the least (-39) or the NF minus $F$ score was largest for the alpha 2 band when stimulated by flicker at $15 \mathrm{~Hz}$ and thus this score was taken as an estimate of the I

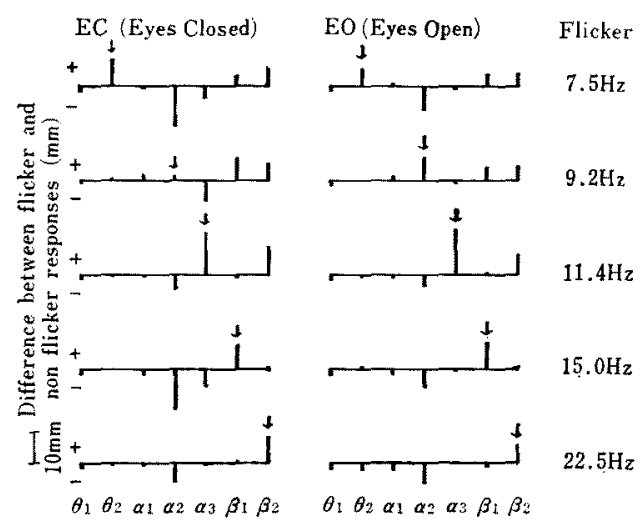

F1G. 3. Differences in occipital integrated values between flicker (F) and nonflicker (NF) conditions or F-NF. Flicker was presented at a frequency shown on the right side, which was included in the band indicated by an arrow. effect for that band. Since his F-NF score for the same band was 13 with flicker at $9.2 \mathrm{~Hz}$ as shown in Fig. 2, the resultant $\mathrm{FE}$ value was estimated at 26 (i.e., -13 $+39)$ by the formula, $\mathrm{FE}=(\mathrm{F}-\mathrm{NF})+\mathrm{I}$.

Fig. 5A shows the means of such $\mathrm{FE}$ values across the 11 subjects with $\mathrm{EC}$ and EO. Although the FE effect appears somewhat less with EO, the difference failed to be significant. Similarly when the same $\mathrm{FE}$ values were plotted for the dominant and the poor alpha subjects as indicated by Fig. 5B, they were higher for the dominant alpha group for all bands but here again the difference was short of statistical significance $(F>1)$. Thus the FE effect may be assumed to be about identical both for the dominant alpha and for the poor alpha subjects. This may be contrasted with the clear differentiation in the spontaneous EEG or NF spectrum between the two groups as shown in Fig. 5C. Second, the FE effect was somewhat larger for alpha 3 than for alpha 2 (Fig. 5B), while the peak component was in the alpha 2 rather than in the alpha 3 band in the NF spectrum (Fig. 5C). In addition, the FE spectral components had a wider distribution than the NF components (Figs. 5 and Fig. 1).

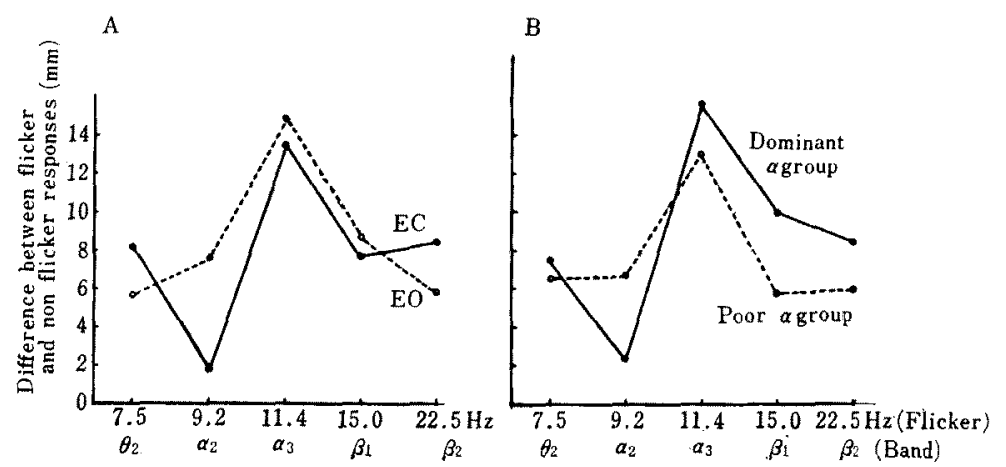

FIG. 4. Difference in integrated values between flicker $(F)$ and nonflicker (NF) conditions with eyes closed (EC) and with eyes opened (EO) (panel A). Panel $B$ indicates the same F-NF differences for 5 dominant alpha and 6 poor alpha subjects who were classified based on the spontaneous NF response. 


\section{Discussion}

As was expected, the occipital EEG activity in terms of integrated values with band-pass filters was reduced by eye opening (EO) not only in alpha but also in theta and beta frequency bands (Fig. 1). This is in accordance with our previous study (Noguchi \& Iwahara, 1971) and similar results were reported with light stimulation (Motokizawa, 1963; Sato \& Mimura, 1956).

There were considerable individual differences both in amplitudes and frequencies of the dominant alpha activity (i.e., the peak component in the NF spectrum) but the blocking effect either by $\mathrm{EO}$ or by flicker stimulation was largest in the frequency band in which the subject's own dominant alpha frequency was included and the overall blocking effect
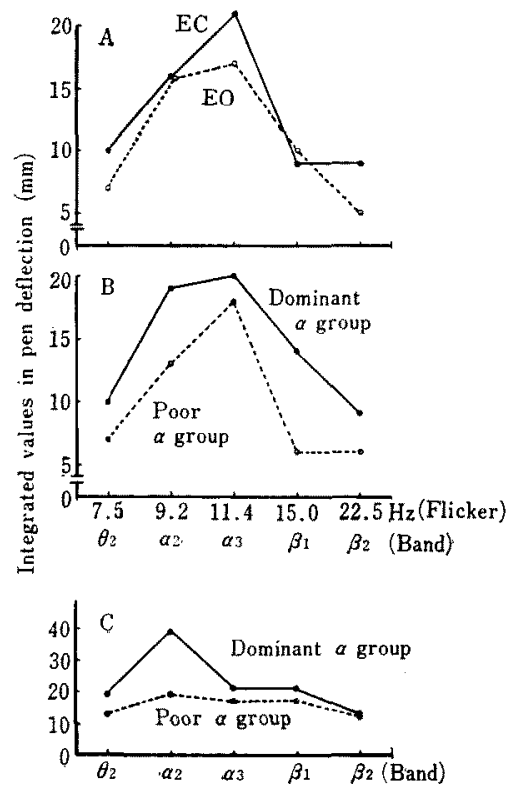

Fig. 5. Panel A shows the frequency-specific effect (FE) with different flicker frequencies when the nonspecific inhibitory effect (I) was ruled out. Panel $B$ indicates the same FE spectrum for the dominant and poor alpha subjects, which may be contrasted with the spontaneous NF spectrum plotted in Panel $\mathrm{C}$. due to EO was relatively independent of the dominant alpha frequency. In addition, the blocking effect due to flicker stimulation as indicated by the negative F-NF score was not dependent upon flicker frequency outside the frequency bands in which the frequency specific driving effect (FE) may be assumed (Fig. 3).

In this study, the occipital activity to flicker stimulation or $\mathrm{F}$ is postulated as a first approximation to be a linear function of the frequency specific effect (FE) and the frequency nonspecific blocking effect (I) or in formula;

$$
\mathrm{F}=\mathrm{NF}+\mathrm{FE}-\mathrm{I},
$$

where NF is the cortical activity without flicker, and both $\mathrm{F}$ and NF values were measured in terms of integrated values with band-pass filters. On the other hand, the I values were estimated from the data and thus the $\mathrm{FE}$ values could be computed by the postulated formula.

When the FE values were plotted as a function of the flicker frequency, the obtained FE spectrum was considerably different from the basic EEG spectrum or the NF spectrum. First, the peak component was in alpha 3 band in the former but in the alpha 2 band in the latter. Second, the spectral components had a wider distribution for the FE than in the NF spectrum. Third, the FE spectrum was little affected by eye opening (EO), while the NF spectrum was greatly reduced and flattened in amplitude with EO (Fig. 5A vs. Fig. 1). Fourth, the FE spectrum was not very different if any, whether the basic alpha activity was dominant (abundant) or poor (Fig. 5B vs. Fig. 5C). This last finding agrees with Toman's (1941) study in which the magnitude of flicker potentials was independent of the amount of alpha rhythm, although his analysis was based on visual inspection. Both of these findings may suggest that the neural mechanisms responsible for the FE spectrum or the photic driving 
effect is not necessarily identical to those that produce the basic EEG activity or the NF spectrum.

On the other hand, Sato, Mimura, Ozaki, Yamamoto, Masuya \& Handa (1957) and Mimura (1958) found a close similarity between the power spectrum based on the cross-correlations of the flicker-induced occipital response and the flicker cycle, and that based on the autocorrelations of the spontaneous NF activity, and argued that the spontaneous EEG is identical in its underlying neural mechanisms to the flicker-induced EEG at least as a first approximation. Although their approach was more elaborated than the present one, it is not clear why the nonspecific I effect was not considered in their study. It was noticed that their components showed a wider distribution in the flicker-induced power spectrum than in the spontaneous one, in accordance with the present study.

Differentiation between the specific $\mathrm{FE}$ effect and the nonspecific I effect was made by Sokolov (1958) in his study of the cortical orienting reflex. However, no systematic work has been made either by him or by others concerning the time process of these effects. Evidently, it may be assumed that FE is a specific reflex and $I$ is a nonspecific orienting reflex. Thus $I$ is expected to be extinguished or completely habituated, while FE is expected to be weakened (habituate) but not extinguished. This reasoning is now being investigated in our laboratory. It will also be examined whether the harmonic response induced by flicker at the basic alpha frequency is reduced by I as is the tuned alpha response.

\section{REFERENCES}

IwAHARA, S., \& Nocuchi, S. 1972 Intra- and inter-subject variations of EEG patterns under varying flickering conditions in normal adults. The Fapanese Journal of Psychology, 43, 86-94. (In Japanese with an English abstract.)

Mimura, K. 1958 On the frequency characteristics of the occipital EEG activities. Brain and Nerve (Tokyo), 10, 397-404. (In Japanese with an English abstract.)

Morgan, C. T. 1965 Physiological psychology. New York: McGraw-Hill.

MorokizAwa, F. 1963 Afrequency-analytic study of alpha-blocking in human EEG. Psychiatria et Neurolgia Japonica, 65, 433-440. (In Japanese with an English abstract.)

Noguchi, S., \& Iwahara, S. 1971 Effects of flicker stimulation upon the EEG under the eye-closed and eye-opened conditions and the eye-closed mental calculation condition. The Japanese Fournal of Psychology, 42, 287295. (In Japanese with an English abstract.)

Sato, K., \& Mimura, K. 1956 The effect of photic stimuli on the constituent element of EEG. Japanese Journal of Physiology, 6, 206-217.

Sato, K., Mimura, K., Ozaki, T., Yamamoto, Y., Masuya, S., \& Honda, N. 1957 On the "transformating action" of the brain shown in the brain wave. Japanese Journal of Physiology, 7, 181-189.

Sokolov, E. N. 1958 Perception and the conditioned reflex. In Japanese translation by Kaneko, T., \& Suzuki, H. 1965 Tokyo: Sekaishoin.

Toman, J. 1941 Flicker potentials and the alpha rhythm in man. Journal of Neurophysiology, 4, 51-61.

(Received Jan. 29, 1973) 